\title{
Fetal Heterotaxy with Tricuspid Atresia, Pulmonary Atresia, and Isomerism of the Right Atrial Appendages at 22 Weeks
}

\author{
Julia E. Solomon, MD ${ }^{1}$ John H. Stock, MD ${ }^{2}$ Randy R. Richardson, MD ${ }^{3}$ Norman H. Silverman, MD
}

${ }^{1}$ Division of Maternal Fetal Medicine, Fetal Diagnostic Center, St. Joseph's Hospital and Medical Center-Dignity Health, Phoenix, Arizona

2 Arizona Pediatric Cardiology Consultants, Pediatrix Medical Group,

Address for correspondence J. E. Solomon, MD, Fetal Diagnostic Center, 1727 W Frye Road, Suite 210, Chandler, AZ 85224 (e-mail: julia.solomon@dignityhealth.org).

Phoenix, Arizon

${ }^{3}$ Department of Radiology, St. Joseph's Hospital and Medical Center-

Dignity Health, Phoenix, Arizona

${ }^{4}$ Division of Pediatric Cardiology, University of California at

San Francisco, San Francisco, California

Am J Perinatol Rep 2013;3:97-102.

\begin{abstract}
Keywords

- fetus

- heterotaxy

- right atrial isomerism

- tricuspid atresia

- echocardiography

- ultrasound

We report the accurate prenatal diagnosis at 22 weeks gestation of right atrial isomerism in association with tricuspid atresia. Several distinctive sonographic features of isomerism of the right atrial appendages were present in this fetus: complex cardiac abnormality, ventriculoarterial discordance, juxtaposition of the aorta and the inferior vena cava to the right side, pulmonary atresia, and anomalous pulmonary venous return to the morphological right atrium. Tricuspid atresia, which is an extremely rare lesion within heterotaxy spectrum disorders, was present. Postnatal investigations confirmed all prenatally diagnosed abnormalities, with additional findings of pulmonary atresia with discontinuous pulmonary arteries and bilateral arterial ducts, asplenia, and bilateral eparterial bronchi. To our knowledge, tricuspid atresia in the setting of isomerism of the right atrial appendages has not previously been diagnosed or reported prenatally. Because of the complexity of cardiac lesions that may be present in cases of atrial isomerism, these disorders should be considered even if sonographic findings are uncommon or atypical.
\end{abstract}

\section{Case Report}

A 31-year-old gravida 1 was seen for routine evaluation of fetal anatomy at 22 weeks. A complex cardiac abnormality was diagnosed. Findings at the time of the detailed exam included levocardia with a normal left-sided stomach bubble, but a midline-appearing liver. An absent right atrioventricular valvar connection typical of tricuspid atresia was identified with a patent and normal-sized left atrioventricular (mitral) valve (-Fig. 1A). A large unrestricted ventricular septal defect was noted, communicating to a relatively hypoplastic right ventri- cle. The atrial septum demonstrated normal bowing of the atrial septum from the right to the left atrium with evidence of flow on color flow Doppler, with a moderate-sized ostium primum atrial septal defect. Atrial morphology was suggestive of bilateral right atrial appendages but inconclusive (-Fig. 1B). There was D-transposition with the aorta arising from the anterior, morphologically right ventricle (-Fig. 1C). The aortic arch was right-sided with mirror image branching. A discrete main pulmonary artery was not identified in the usual medial position; however, retrograde flow was noted in the ductus arteriosus both in two-dimensional imaging and received

February 26, 2013 accepted after revision

March 16, 2013

published online

May 21, 2013
DOI http://dx.doi.org/ 10.1055/s-0033-1344004. ISSN 2157-6998.
Copyright (c 2013 by Thieme Medical Publishers, Inc., 333 Seventh Avenue, New York, NY 10001, USA. Tel: +1(212) 584-4662.
License terms

(요 (1) $\Theta \circledast$ 

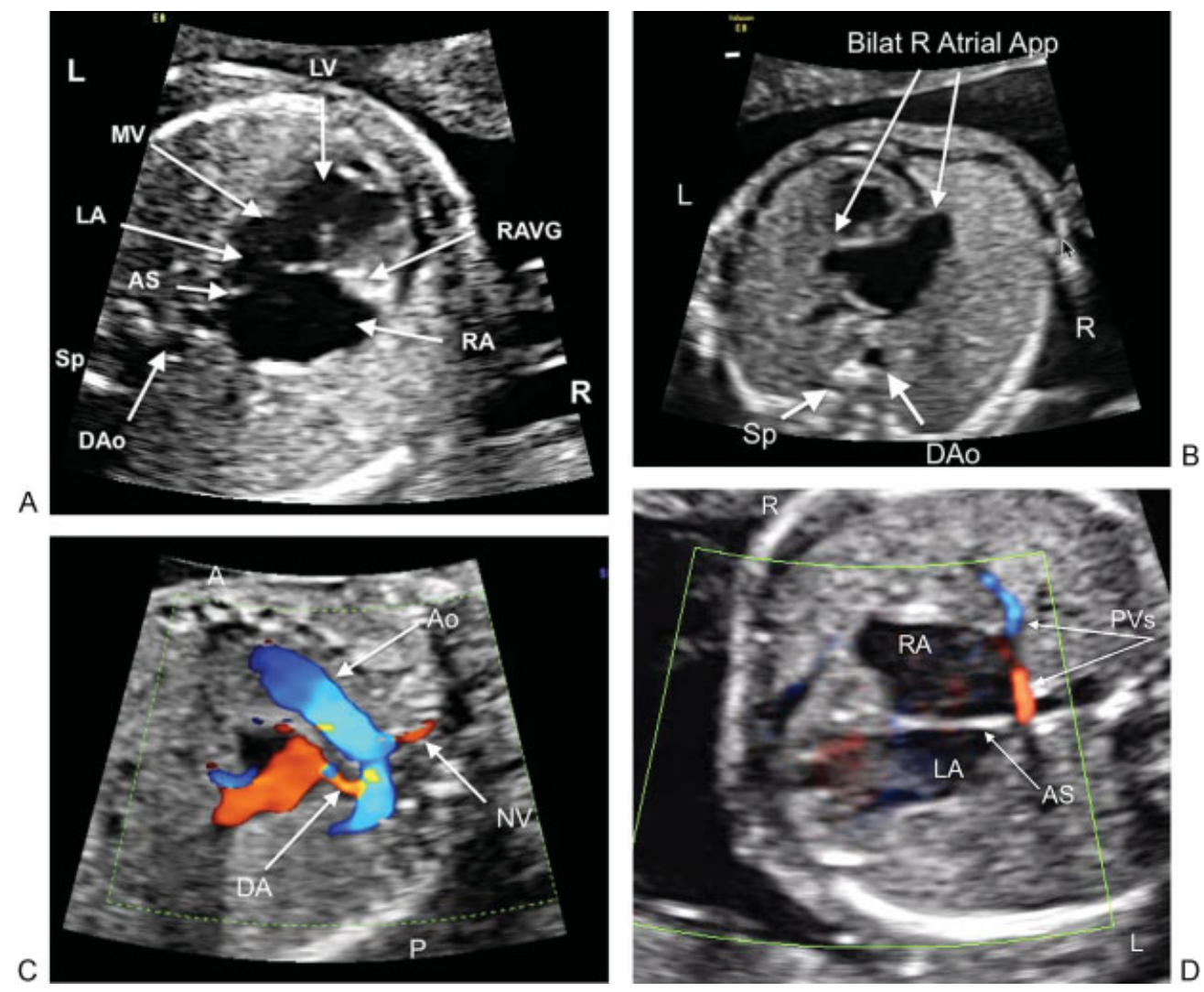

Fig. 1 Prenatal (fetal) ultrasound images. (A) Four-chamber view demonstrating levocardia with tricuspid atresia and a large unrestricted ventricular septal defect. (B) Four-chamber view demonstrating atrial morphology. Arrows point to bilateral atrial appendages that appear morphologically right-sided. (C) Arch view of D-transposed anterior aorta, demonstrating neck vessels and retrograde flow into the ductus arteriosus (arrows). (D) Pulmonary veins returning to the right atrium, with atrial septum well visualized. Abbreviations: Ao, aorta; AS, atrial septum; Bilat, bilateral; Bilat R Atrial App, bilateral right atrial appendages; DA, ductus arteriosus; DAo, descending aorta; L, left; LA, left atrium; LV, left ventricle; MV, mitral valve; NV, neck vessel; P, posterior; PVs, pulmonary veins; R, right; RA, right atrium; RAVG, right atrioventricular groove; Sp, spine.

four-dimensional spatiotemporal image correlation imaging, both with superimposed color flow Doppler, consistent with presumptive pulmonary atresia. Bilateral superior venae cavae were also identified, without evidence of a bridging innominate vein. All four pulmonary veins were seen to drain into the right atrium without obstruction ( - Fig. 1D). Finally, assessment of the abdominal vessel configuration demonstrated both an aorta and inferior vena cava that were rightsided. Based on the presence of a complex cardiac malformation and the latter two findings of total anomalous pulmonary venous return and juxtaposition of both the aorta and inferior vena cava to the right side, our prenatal diagnosis was that of isomerism of the right atrial appendages.

Formal karyotype testing with microarray was offered to the parents and declined. The pregnancy progressed otherwise uneventfully with normal interval growth and reassuring parameters of fetal well-being. The patient labored spontaneously at $39^{1 / 7}$ weeks; the fetus was delivered vaginally with a birth weight of 3,430 $\mathrm{g}$ and Apgar scores of 8 at 1 minute and 9 at 5 minutes. Postnatal prostaglandin infusion was initiated and an echocardiogram was performed, which confirmed of all of the prenatal ultrasound findings ( $\mathbf{- F i g . 2}$ ). The patent atrioventricular valve was confirmed to be a mitral valve morphologically, without evidence of a septal papillary muscle. Ventricular walls were predominately smooth, again consistent with left ventricular morphology. Cardiac catheterizations confirmed a right-sided aorta ( - Fig. $\mathbf{3}$ ) and

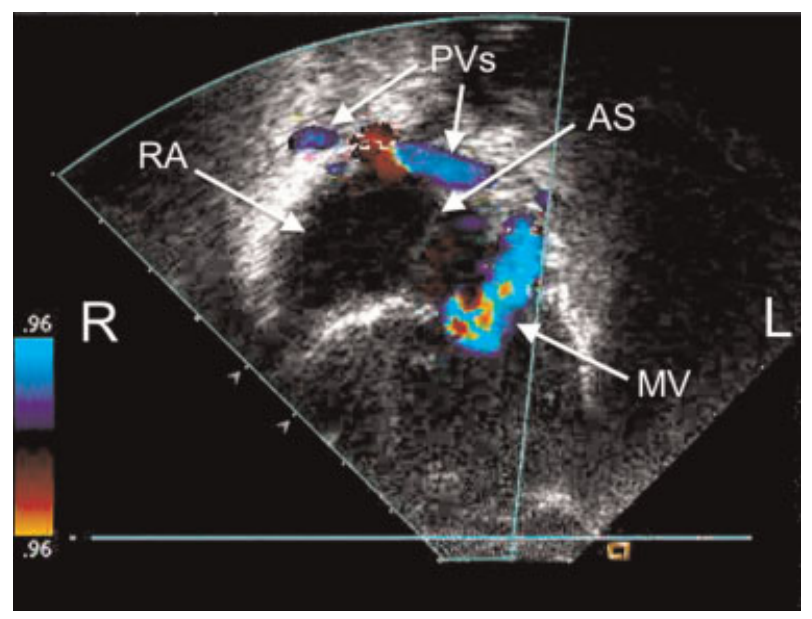

Fig. 2 Postnatal echocardiogram, apical view, confirming tricuspid atresia, pulmonary veins returning to the right atrium, and regurgitation through the mitral valve. Abbreviations: AS, atrial septum; L, left; MV, mitral valve; PVs, pulmonary veins; $R$, right; $R A$, right atrium. 


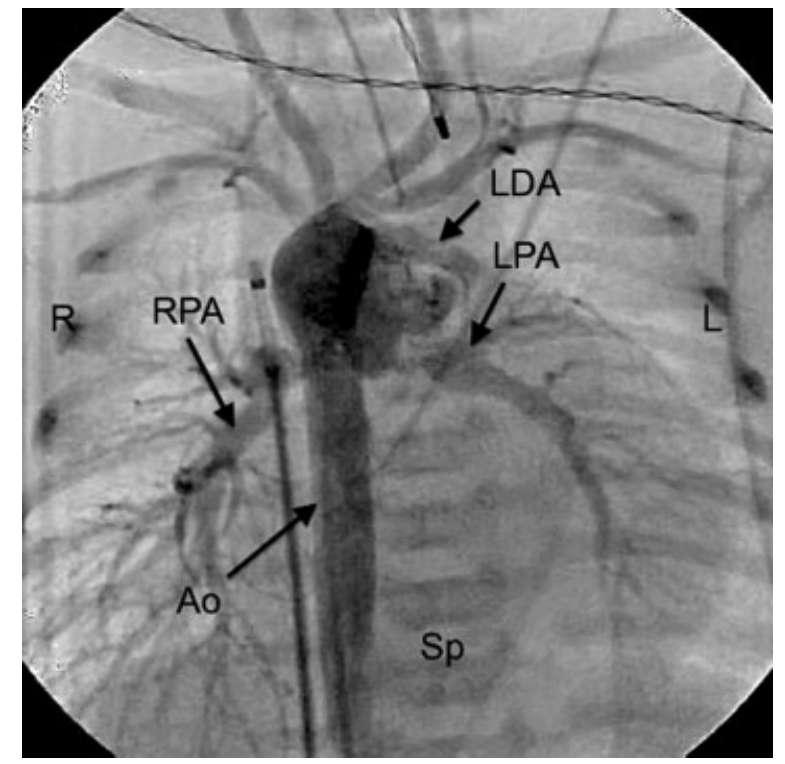

Fig. 3 Postnatal aortogram (anterior-posterior view), demonstrating a right-sided aorta with mirror image branching and discontinuous pulmonary arteries. Abbreviations: Ao, aorta; L, left; LDA, left ductus arteriosus; LPA, left pulmonary artery; R, right; RPA, right pulmonary artery; Sp, spine.

discontinuous pulmonary arteries arising from bilateral arterial ducts.

Both abdominal ultrasonography and computed tomography scan failed to identify a spleen in the usual or any other location. Barium studies did not identify any malrotation abnormalities of the gut. Cardiac computerized tomographic angiography with three-dimensional reconstruction also confirmed the echocardiographic findings ( $\mathbf{- F i g . 4 A}$ ). Origin of the pulmonary arteries/ducts was the brachiocephalic trunk for the left side and the inferior aspect of the aortic arch for the right side ( $\mathbf{F i g}$. 4B). Imaging of the bronchial tree was conclusive for bilateral eparterial (right-sided) bronchi

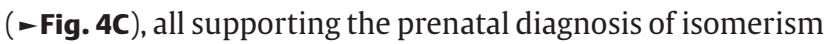
of the right atrial appendages.

The neonate underwent bilateral ductal-pulmonary artery stenting at 8 days of life. Bilateral bidirectional cavopulmonary shunts were performed at 4 months of age. The child is currently 11 months of age and thriving. There has been no postnatal evidence of associated dysmorphology or other unrelated abnormalities, and no further genetic testing was performed.

\section{Discussion}

Visceroatrial heterotaxy syndrome is a complex developmental condition that is frequently associated with serious congenital cardiac disease. This set of disorders is typically characterized by abnormal arrangement of cardiac and visceral organs across a left-right axis, different from situs solitus or complete situs inversus. Historically, heterotaxy has been divided into the subcategories of right or left isomerism based on the appearance of the atrial appendages; isomerism refers to the symmetric development of structures that are normally asymmetric. ${ }^{1}$ More recently, the extent of pectinate muscle proximity to the atrioventricular valves has been found to be more accurate in assessing right- or leftsidedness than atrial appendage morphology, ${ }^{2}$ but this cannot be defined by pre- or postnatal echocardiography. Isomerism of the right atrial appendages has classically been associated with asplenia and isomerism of the left atrial appendages with polysplenia. However, spleen status may be discordant in either right or left isomerism, ${ }^{3}$ and splenic anatomy is less well correlated with atrial appendage arrangement than bronchopulmonary anatomy, which is highly consistent. ${ }^{1}$

Both right and left atrial isomerism are associated with complex congenital cardiac defects in addition to situs abnormalities of thoracic and abdominal organs. With the exception of stomach bubble and gall bladder laterality, abdominal visceral situs can be difficult to assess in a fetus.
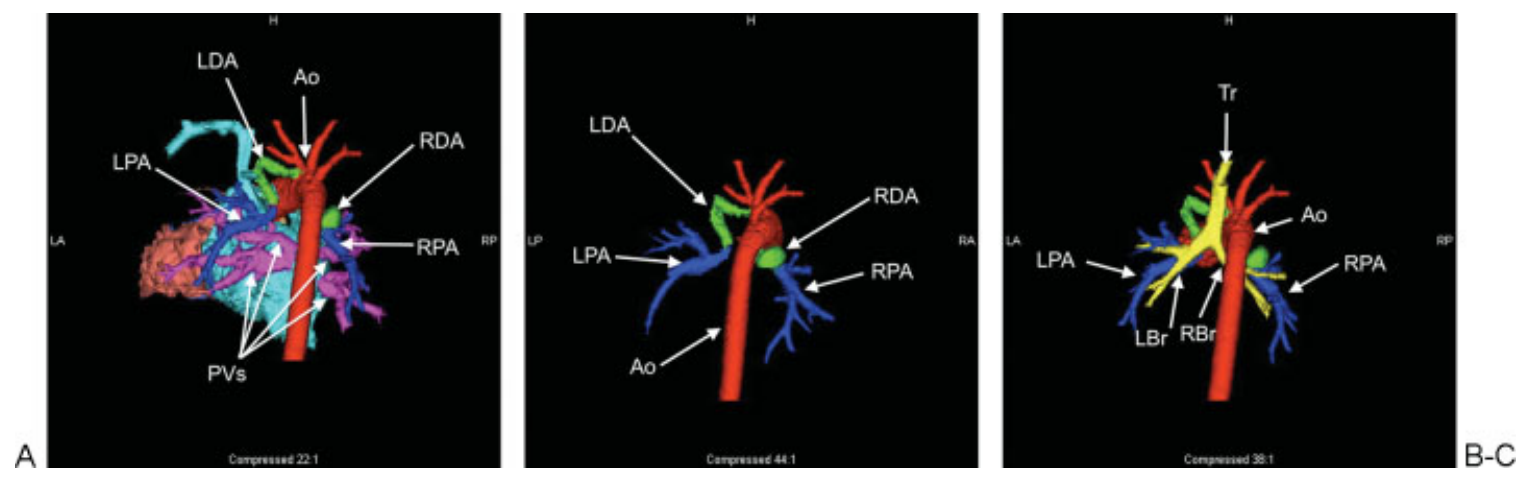

Fig. 4 Cardiac computerized tomographic angiogram with three-dimensional reconstruction. (A) Posterior view of the heart and right-sided aortic arch; all four pulmonary veins (pink) return discretely to the right atrium. (B) Posterior aortic arch view demonstrating discontinuous pulmonary arteries (blue) arising from bilateral ducts (green). The right pulmonary artery/duct arises from the underside of the aortic arch; the left pulmonary artery/duct arises from the brachiocephalic trunk. (C) Posterior view of the aortic arch and pulmonary arteries demonstrating bilateral eparterial bronchi (yellow). Abbreviations: Ao, aorta; LA, left anterior; LBr, left mainstem bronchus; LDA, left ductus arteriosus; LP, left posterior; LPA, left pulmonary artery; PVs, pulmonary veins; RA, right anterior; RBr, right mainstem bronchus; RDA, right ductus arteriosus; RP, right posterior; RPA, right pulmonary artery; Tr, trachea. 
In a small case series, color flow Doppler identification of a splenic artery has been successful in documenting a normally located spleen but can represent a false-negative in cases of multiple small spleens. ${ }^{4}$ In the absence of pleural effusions, lung lobation can be extremely hard to assess. Because the bronchial tree is fluid-filled in the fetus, bronchopulmonary situs may potentially be determined by ultrasound, but this is time-consuming and subject to limitations of fetal positioning and is therefore infrequently possible. ${ }^{5}$ Intestinal malrotation is not amenable to prenatal ultrasound diagnosis.

Because of the frequent segregation of often-typical cardiac abnormalities in either form of isomerism of the atrial appendages, the correct identification of these findings affords the prenatal sonologist the best opportunity at an antenatal diagnosis. Ultrasound assessment of fetal atrial appendage shape has been performed correctly in a small series, but remains difficult and not completely reliable. ${ }^{6}$ Because of the differential flow and pressure characteristics noted during fetal life, isomeric appendages may also be dissimilar in size and shape, making the diagnosis difficult. ${ }^{7}$ The pectinate muscles cannot be evaluated at all. Aside from analysis of segmental anatomy, knowledge of the associations of intracardiac morphology and extracardiac findings affords the best opportunity to arrive at a correct diagnosis in these syndromes.

In cases of isomerism of the right atrial appendages, there is substantial literature devoted to the documentation of the specific malformations. ${ }^{8-12}$ These include abnormalities of cardiac position, systemic and pulmonary venous return, forms of atrioventricular canal defects, abnormalities of ventriculoarterial connections, and arterial atresias or stenoses. Some of these malformations are extremely prevalent in the syndrome of right atrial isomerism, many of which are noted in this fetus. These include abnormal ventriculoarterial connections, pulmonary artery outflow obstruction, bilateral superior venae cavae, juxtaposition of the aorta and inferior vena cava, anomalous pulmonary venous return, and an absent coronary sinus. The presence of numerous abnormalities typical for the disorder allowed us to counsel the parents accurately.

There were several uncommon features. First, this fetus demonstrated anomalous pulmonary venous return directly to the right atrium. In most cases of isomerism of the right atrial appendages, anomalous pulmonary venous return is supra- or infracardiac. ${ }^{2,11}$ The combination of bilateral systemic venous return and anomalous pulmonary venous return to the right-sided atrium was seen in only $10 \%$ of cases in a large series of right atrial isomerism. ${ }^{2}$

Second, the atrioventricular connection is extremely atypical. In up to $90 \%$ of cases of right atrial isomerism, this connection is in the form of a complete atrioventricular canal defect. ${ }^{9}$ In our patient, tricuspid atresia with a patent mitral valve is noted. To our knowledge, there has only been one prior reported case of tricuspid atresia associated with fetal right atrial isomerism, and this specific abnormality was only diagnosed postnatally. ${ }^{10}$ In fact, the two largest fetal series of tricuspid atresia (totaling 142 patients) did not identify a single case that was associated with a heterotaxy abnormality, even when postnatal investigations were considered. ${ }^{13,14}$ Our case represents the first accurate prenatal diagnosis of tricuspid atresia on a background of right atrial isomerism. Because of this rare association, the larger diagnosis of isomerism of the right atrial appendages could be potentially dismissed, leading to suboptimal counseling regarding outcome and prognosis. With careful evaluation and documentation of the features in this case that are highly typical of the disorder, the correct diagnosis was made.

The implications of isomerism of the right atrial appendages extend beyond the complexity of the cardiac abnormality itself. Most children are functionally asplenic and have a lifelong susceptibility to encapsulated bacteria. ${ }^{15}$ Despite advances in surgical intervention, the mortality rate for patients requiring surgical intervention in the neonatal period is $75 \%$, with those requiring pulmonary vein repair having a surgical mortality of $95 \%{ }^{9}$

Our diagnosis confirms that recognition of this constellation of abnormalities permits accurate prenatal diagnosis and counseling even when atypical findings are present.

\section{References}

1 Jacobs JP, Anderson RH, Weinberg PM, et al. The nomenclature, definition and classification of cardiac structures in the setting of heterotaxy. Cardiol Young 2007;17(Suppl 2):1-28

2 Uemura H, Ho SY, Devine WA, Kilpatrick LL, Anderson RH. Atrial appendages and venoatrial connections in hearts from patients with visceral heterotaxy. Ann Thorac Surg 1995;60:561-569

3 Anderson C, Devine WA, Anderson RH, Debich DE, Zuberbuhler JR. Abnormalities of the spleen in relation to congenital malformations of the heart: a survey of necropsy findings in children. $\mathrm{Br}$ Heart J 1990;63:122-128

4 Abuhamad AZ, Robinson JN, Bogdan D, Tannous RJ. Color Doppler of the splenic artery in the prenatal diagnosis of heterotaxic syndromes. Am J Perinatol 1999;16:469-473

5 Yoo SJ, Friedberg MK, Jaeggi E. In: Abnormal visceral and atrial situs and congenital heart disease. Yagel S, Silverman $\mathrm{NH}$, Gembruch U, eds. Fetal Cardiology. 2nd ed. Informa Healthcare USA Inc. 52 Vanderbilt Avenue New York, NY 2009:352-354

6 Berg C, Geipel A, Kohl T, et al. Fetal echocardiographic evaluation of atrial morphology and the prediction of laterality in cases of heterotaxy syndromes. Ultrasound Obstet Gynecol 2005;26:538-545

7 Van Praagh R, Van Praagh S. Atrial isomerism in the heterotaxy syndromes with asplenia, or polysplenia, or normally formed spleen: an erroneous concept. Am J Cardiol 1990;66:15041506

8 Ho SY, Cook A, Anderson RH, Allan LD, Fagg N. Isomerism of the atrial appendages in the fetus. Pediatr Pathol 1991;11:589-608

9 Hashmi A, Abu-Sulaiman R, McCrindle BW, Smallhorn JF, Williams WG, Freedom RM. Management and outcomes of right atrial isomerism: a 26-year experience. J Am Coll Cardiol 1998;31:1120-1126

10 Berg C, Geipel A, Kamil D, et al. The syndrome of right isomerismprenatal diagnosis and outcome. Ultraschall Med 2006;27: 225-233

11 Rubino M, Van Praagh S, Kadoba K, Pessotto R, Van Praagh R. Systemic and pulmonary venous connections in visceral heterotaxy with asplenia. Diagnostic and surgical considerations based on seventy-two autopsied cases. J Thorac Cardiovasc Surg 1995; 110:641-650 
12 Taketazu M, Lougheed J, Yoo SJ, Lim JS, Hornberger LK. Spectrum of cardiovascular disease, accuracy of diagnosis, and outcome in fetal heterotaxy syndrome. Am J Cardiol 2006;97:720-724

13 Berg C, Lachmann R, Kaiser C, et al. Prenatal diagnosis of tricuspid atresia: intrauterine course and outcome. Ultrasound Obstet Gynecol 2010;35:183-190
14 Wald RM, Tham EB, McCrindle BW, et al. Outcome after prenatal diagnosis of tricuspid atresia: a multicenter experience. Am Heart J 2007;153:772-778

15 Cohen MS, Anderson RH, Cohen MI, et al. Controversies, genetics, diagnostic assessment, and outcomes relating to the heterotaxy syndrome. Cardiol Young 2007;17(Suppl 2):29-43 